\title{
Causation in epidemiology
}

\author{
M Parascandola, D L Weed
}

Abstract

Causation is an essential concept in epidemiology, yet there is no single, clearly articulated definition for the discipline. From a systematic review of the literature, five categories can be delineated: production, necessary and sufficient, sufficient-component, counterfactual, and probabilistic. Strengths and weaknesses of these categories are examined in terms of proposed characteristics of a useful scientific definition of causation: it must be specific enough to distinguish causation from mere correlation, but not so narrow as to eliminate apparent causal phenomena from consideration. Two categoriesproduction and counterfactual-are present in any definition of causation but are not themselves sufficient as definitions. The necessary and sufficient cause definition assumes that all causes are deterministic. The sufficient-component cause definition attempts to explain probabilistic phenomena via unknown component causes. Thus, on both of these views, heavy smoking can be cited as a cause of lung cancer only when the existence of unknown deterministic variables is assumed. The probabilistic definition, however, avoids these assumptions and appears to best fit the characteristics of a useful definition of causation. It is also concluded that the probabilistic definition is consistent with scientific and public health goals of epidemiology. In debates in the literature over these goals, proponents of epidemiology as pure science tend to favour a narrower deterministic notion of causation models while proponents of epidemiology as public health tend to favour a probabilistic view. The authors argue that a single definition of causation for the discipline should be and is consistent with both of these aims. It is concluded that a counterfactually-based probabilistic definition is more amenable to the quantitative tools of epidemiology, is consistent with both deterministic and probabilistic phenomena, and serves equally well for the acquisition and the application of scientific knowledge.

(F Epidemiol Community Health 2001;55:905-912)

Office of Preventive

Oncology, National

Cancer Institute,

Executive Plaza South,

Suite T-41, 6120

Executive Blvd, MSC

7105, Bethesda, MD

20892-7105, USA

Correspondence to:

Dr Parascandola

(paramark@mail.nih.gov)

Accepted for publication 22 May 2001

"The view [of causation] we adopt has consequences which reach far beyond informal discussion during coffee breaks."1

Causation is an essential concept in the practice of epidemiology. Causal claims like "smoking causes cancer" or "human papilloma virus causes cervical cancer" have long been a standard part of the epidemiology literature.
But despite much discussion of causes, it is not clear that epidemiologists are referring to a single shared concept.

Multiple definitions of cause have been offered in epidemiology. In 1970 MacMahon and Pugh wrote that "the word cause is an abstract noun and, like beauty, will have different meanings in different contexts." ${ }^{2}$ Yet cause is also a scientific term, and it is important that there is some common thinking among epidemiologists about what is meant in saying " $\mathrm{X}$ causes Y." In practice many causal statements are ambiguously stated; for example, "smoking is a cause of cancer" may mean "every smoker will develop cancer" or it can be construed as "at least one smoker will develop cancer," depending upon the underlying concept of causation the speaker has in mind.

There have been calls in the recent epidemiological literature for more attention to the theoretical and conceptual basis of epidemiology. ${ }^{4}$ In this journal, Nijhuis and Van der Maesen urged readers to investigate the philosophical foundations of public health, with special attention to ontological concerns (for example, the nature of basic concepts such as "public" and "health"). ${ }^{6}$ In this paper, we focus on causation.

This paper reviews the epidemiological literature, seeking patterns and dominant definitions. The strengths and weaknesses of different approaches to defining causation are examined, drawing also on the rich philosophical literature on this topic. From this, a key distinction is drawn between deterministic and probabilistic concepts of causation. The choice between these two approaches is in part driven by researchers' views about the role of epidemiology in relation to other sciences and to public health. We explain this relation and make a recommendation about what type of causal definition best meets the goals of the discipline of epidemiology.

The focus of attention in this paper will be on chronic disease epidemiology. It is in this area that the concept of causation has proved most elusive and has provoked the most confusion and debate. The focus will also be on the ontological nature of causation rather than on causal inference, a more epistemological concern; it is this conceptual understanding that those who call for more theory have noted is deficient.

\section{Literature review}

We searched for definitions of causation in the epidemiological literature to determine how epidemiologists characterise causes. Heuristic devices that are not strictly definitions, such as the "web of causation", were excluded. ${ }^{7}$ The web is not intended to be a theory or to provide causal explanations, but merely to act as a 
metaphor for the idea that causal pathways are complex and interconnected. ${ }^{8}$

The MEDLINE database was searched using PUBMED. The English language literature from 1990 to August 1999 was searched for MeSH terms "causality" and either "epidemiology" or "epidemiologic methods." The terms were used unexploded so that subheadings of these terms would be excluded. Altogether 148 records were retrieved. "Causality" was only introduced as a MeSH heading in 1990. The literature from 1966 through 1989 was searched for "logic" and "epidemiology" as keywords. A search for "causation" and "epidemiology" during this period also revealed some further references. Articles also were identified through the authors' collections of papers on the topic of causation in epidemiology. The reference lists of review articles on causation in epidemiology were perused for mention of additional relevant articles. Major epidemiology textbooks were also reviewed for discussions of causation. From among these sources, at least five distinct definitions can be identified.

\section{Making sense of multiple definitions}

After reviewing the literature, we have found that cause is defined in the following ways: production, necessary causes, sufficientcomponent causes, probabilistic causes, and counterfactuals (table 1). Interestingly, these five types of definitions correspond with major approaches to causation found in the philosophical literature. However, discussion of these definitions in the epidemiological literature fails to recognise some important distinctions and criticisms from the philosophical literature. In particular, it is important to distinguish between deterministic and probabilistic causes and between ontological definitions of causation and causal models used in scientific inference. While these five definitions are not all mutually exclusive, there are significant consequences to choosing one definition over another.

\section{PRODUCTION}

Under this definition, a cause is something that, in short, creates or produces an effect. A similar notion is that causes affect outcomes (page 637$)^{9}$ or that causes alter outcomes. ${ }^{2}$ In contrast, a non-causal association does not involve production; A coincides with $\mathrm{B}$, but $\mathrm{A}$ does not produce $B$. But while the notion of production draws an ontological distinction between causal and non-causal associations, the definition is vague about what "production" or "creation" means. Thus, the elusive concept of causation is defined only in terms of another equally elusive concept. Philosophers have long been aware of the weaknesses inherent in this definition of causation; David Hume and Bertrand Russell both reject the notion of causation because it retains this mysterious element. ${ }^{11}{ }^{11}$ The shortcomings of this definition have also been noted in the epidemiological literature. ${ }^{12} 13$ Thus, a more robust definition is needed.

\section{NECESSARY CAUSES}

Many discussions of causation in epidemiology incorporate the concepts of necessary and sufficient causes. Succinctly, a necessary cause is a condition without which the effect cannot occur, and a sufficient cause is a condition with which the effect must occur.(page 191-2) ${ }^{14}$ (page $4-5)^{15}(\text { page } 326-7)^{16}$ (page 21$)^{17}$ (page $261)^{18}$ (page 27) ${ }^{19}$ (page $\left.45-47\right)^{20}$ Four different types of causal relations can be derived from these two definitions: necessary and sufficient, necessary but not sufficient, sufficient but not necessary, and neither necessary nor sufficient.

A small minority of epidemiologists maintain that the term "cause" should be limited to highly specific necessary conditions. ${ }^{21}{ }^{22}$ The view that all causes must be necessary for their effects is traditionally associated with the germ theory of disease, wherein each disease-for example, tuberculosis - is caused by a specific infectious agent-for example, tubercle bacillus. ${ }^{23}$ Stehbens applies this model to all diseases, claiming that no particular disease has been proven to have more than one cause. Instead, says Stehbens, multi-causal models merely indicate gaps in scientific understanding, as scientists have not yet uncovered a sole, specific cause or adequately defined a disease outcome. Thus, for example, a high cholesterol diet cannot be called a cause of heart disease. ${ }^{21}$ Charlton similarly claims that the basic sciences are built on the concept of necessary causes and that epidemiology, in order to be scientific, should follow that model. ${ }^{22}$

Support for this narrow definition of causation is ultimately a product of the lingering historical influence of scientific determinism. Since Galileo and Newton, classical physics has been rooted in a world view in which complex phenomena can always be reduced to simple, deterministic mechanisms. Strict determinism requires a one to one correspondence between cause and effect; the same cause invariably leads to the same effect, with no role for chance or stochastic variation. In the biomedical

Table 1 Definitions of causation from the epidemiological literature

\begin{tabular}{ll}
\hline $\begin{array}{l}\text { Production } \\
\text { Necessary causes }\end{array}$ & $\begin{array}{l}\text { Causes are conditions that play essential parts in producing the occurrence of disease. }{ }^{29} \\
\text { A necessary cause is a condition without which the effect cannot occur. For example, HIV infection is a } \\
\text { necessary cause of AIDS. }{ }^{14-22}\end{array}$ \\
$\begin{array}{l}\text { Sufficient-component } \\
\text { causes }\end{array}$ & $\begin{array}{l}\text { A sufficient cause guarantees that its effect will occur; when the cause is present, the effect must occur. } \\
\text { A sufficient-component cause is made up of a number of components, no one of which is sufficient on } \\
\text { its own but which taken together make up a sufficient cause. }{ }^{26}{ }^{29} \\
\text { Probabilistic cause } \\
\text { A probabilistic cause increases the probability of its effect occuring. }{ }^{112}{ }^{15} 19{ }^{35-37} \text { Such a cause need not be } \\
\text { either necessary or sufficient. } \\
\text { A counterfactual cause makes a difference in the outcome (or the probability of the outcome) when it is } \\
\text { present, compared with when it is absent, while all else is held constant. }{ }^{46-48} \text { The counterfactual } \\
\text { approach also does not specifically require that causes must be necessary or sufficient for their effects. }\end{array}$ \\
\hline
\end{tabular}


context, 19th century physiologist Claude Bernard argued that for medicine to be scientific, like physics or chemistry, it must recognise only causes that operate through specific and deterministic biological mechanisms (of course, Bernard was writing before the 20th century probabilistic revolution in physics). ${ }^{24}$

But epidemiologists and pathologists have, at least since the advent of the chronic disease era, acknowledged that not all diseases can be attributed to a single necessary cause. ${ }^{25}$ Cigarette smoke, for example, is not necessary for development of lung cancer. In fact, some epidemiology texts and commentaries have stated that causes of complex chronic diseases, like cancer and heart disease, tend to fit into the "neither necessary nor sufficient" category. (page 175) ${ }^{14}$ (page 5) $)^{15}$ (page 46) ${ }^{20}$ Medicine is full of such relations. Moreover, the arguments of Charlton and Stehbens are circular; they argue that science has never identified nonspecific, unecessary causes because they exclude such causes by definition. In sum, while some causes may be necessary for their effects, this formulation is inadequate as a definition of causation.

SUFFICIENT-COMPONENT CAUSES

The sufficient-component cause definition, articulated by Rothman, ${ }^{26}$ improves upon the necessary cause view presented above by admitting causes that are neither specific nor strictly necessary for their effects. A sufficientcomponent cause is made up of a number of components, no one of which is sufficient for the disease on its own. When all the components are present, however, a sufficient cause is formed. Because more than one set of components may be sufficient for the same effect, a disease may have multiple causes. This definition is essentially identical to an influential account in the philosophical literature introduced by John Stuart Mill ${ }^{27}$ and more recently promoted by Mackie. ${ }^{28}$ While in practice scientists and public health practitioners may focus their interest on a particular component of a sufficient cause (such as tobacco advertising), the true cause of a disease, according to this view, is the total assemblage of conditions that are together sufficient for the disease.

But, like the necessary cause definition, the sufficient-component cause definition retains an assumption of scientific determinism that often goes unacknowledged. There are, in fact, two ways in which a cause can be necessary for some effect: (1) it can be necessary in any set of circumstances (the tubercle bacillus is necessary for any case of tuberculosis) or (2) it can be necessary only in a particular set of circumstances in which no other sufficient causes are present (uranium exposure is not a necessary precursor for lung cancer, but perhaps for a particular non-smoking uranium miner his radiation exposure was necessary for his lung cancer to develop when it did). The sufficientcomponent cause definition includes causes that are not necessary in the former sense, but requires that, within a given set of circumstances, all causes must be necessary and sufficient. For the individual, fatalism prevails: "these risks are either one or zero, according to whether the individual will or will not get lung cancer."(page 9) ${ }^{29}$ Thus, all events are wholly "deterministic" because their occurrence or non-occurrence is completely determined by the existing circumstances. While few epidemiologists explicitly subscribe to a strict determinism, the fact that this principle underlies Rothman's widely cited account is noteworthy.

What is the problem with a principle of determinism? Few causes that epidemiologists (or other biomedical scientists) identify demonstrate a pattern of one to one correspondence with their effects. Smokers develop more lung cancers, but smoking is not by itself necessary or sufficient for developing lung cancer. The sufficient-component cause definition postulates that smoking is one element in a sufficient cause and that the other elements simply have not been identified yet. But this is a strong belief to hold in the absence of empirical evidence. In short, the sufficientcomponent cause definition requires that we assume the existence of countless hidden effect modifiers to turn every less than perfect correlation into pure determinism. Moreover, because many causes identified via epidemiology are weak, we would be required to assume that many of these hidden effect modifiers exert effects far stronger than those of known, observable causes. No doubt numerous unknown effect modifiers do exist, but to assume (absent evidence) that they must exist in every corner of the natural world is not a minor presumption. Is such a global assumption biologically plausible? Scientists have long noted that biological processes frequently fail to behave deterministically. ${ }^{30} 31$

The sufficient-component cause definition also has difficulty in generating models that capture the dynamics of cause and effect relations. Because a sufficient cause is fully sufficient for its effect, it cannot readily explain how changes in the quantity of a component cause (say, dose of an antibiotic) could lead to a corresponding change in the effect (say, success in breaking up an infection). Rothman ${ }^{26}$ responds to this concern by positing a set of sufficient causes, each including a different dose of the varying component cause (the antibiotic). The problem with that response is that it still fails to capture dose-response relations as a continuum rather than a series of discrete steps. It is also ontologically unwieldy, introducing unnecessary complexity to a phenomenon more easily explained by other means (as we discuss in the following section) solely to preserve the sufficient-component cause view. By extension, as noted in the literature, similar worries apply to the view's treatment of interaction. ${ }^{32-34}$ In sum, the sufficientcomponent cause view has substantial drawbacks as a definition of causation for epidemiology.

\section{PROBABILISTIC CAUSATION}

Some commentators and textbooks have provided a "probabilistic" or "statistical" definition of causation, ${ }^{112} 1519$ 35-37 where a cause increases that probability (or chance) that its 
effect will occur. For example, one source defines "a cause of cancer as a factor that increases the probability that cancer will develop in an individual. ${ }^{37}$ Under this definition, the occurrence of cancer in an individual may be in part a matter of chance (that is, it is a "stochastic" or "indeterministic" process). Thus, a probabilistic cause may be neither necessary nor sufficient for disease. However, the definition also does not exclude necessary and sufficient causes; a sufficient cause is simply one that raises the probability of its effect occurring to 1 , and a necessary cause raises that probability from 0 .

Probabilistic causation offers an alternative to the determinism inherent in necessary and sufficient causation. In recent years, philosophers of science have developed sophisticated theories of probabilistic causation to explain apparently indeterministic processes. ${ }^{38-44}$ These theories provide the means to construct models of interaction and dose-response relations in quantitative terms through a continuum of probability values. ${ }^{42}$ For example, the relation between dose and, say, the probability of the effect occurring can be described mathematically. Thus, these probabilistic theories provide a better fit to existing epidemiologic tools for effect measurement.

A probabilistic definition of causation is more inclusive than the sufficient-component cause definition; while necessary and sufficient causes can be described in probabilistic terms, probabilistic causes cannot be described in deterministic language. Additionally, the probabilistic definition makes fewer biological assumptions, as it does not require believing in countless hidden effect modifiers for every less than perfect correlation. Science aims to make as few assumptions as possible, and it is contrary to that spirit to assume (by definition) that there can be no probabilistic causes.

A simple fix allows Rothman's "pie" charts for sufficient-component causes ${ }^{26}$ to be used in representing complex probabilistic causes as well. Simply think of the components as contributing together to the probability of the effect, rather than being sufficient for it. If one component is missing, the probability is decreased. Rothman and Greenland ${ }^{29}$ themselves suggest a fix to account for stochastic phenomena. They suggest that the presence or absence of one component might be determined by a random process; yet on their approach the relation between cause and effect remains deterministic, but simply more difficult to predict.

While probabilistic definitions have appeared in the epidemiological literature, there has been little discussion of their strengths and weaknesses or how they relate to other definitions. One concern likely to be raised against a probabilistic definition is that it fails to explain why some smokers develop cancer and others do not, and that is what the "whole discipline of epidemiology" is after.(page 205) ${ }^{1}$ Probability is therefore a "euphemism for ignorance." However, this concern can be
KEY POINTS

- Multiple definitions of causation exist in the epidemiologic literature, but some prominent accounts rely on unfounded biological assumptions.

- A probabilistic definition of causation, along with the notion of counterfactuals, avoids these assumptions and offers practical advantages for epidemiology.

- Exclusive attention to events at the molecular level has been associated with an overly restrictive definition of causation.

- Requirements for an effective definition of causation should be distinguished from those for constructing a useful causal model in a particular investigation.

answered by noting that a probabilistic definition allows for the possibility that other undiscovered causes may also be at work. A newly discovered genetic factor might reveal that smoking dramatically increases lung cancer risk for some individuals but has only a moderate effect on others. There is no reason to assume that a cause increases each individual's risk by the same amount. In fact, because of the mathematical continuum of probability, the probabilistic model allows for a greater range of possible effects. Risks for individuals might differ in more ways than simply being equal to a probability of either 1 or 0 .

Another concern is that the definition remains unclear about what it means to say smoking raises one's probability of developing lung cancer. For example, statisticians Cox and Holland $^{45}{ }^{46}$ both object to a prominent philosophical account of probabilistic causation ${ }^{38}$ on these grounds. They argue that a definition of causation based on statistical inequalities (that is, the probability of the effect is different when the cause is present than when it is absent) is inadequate. Olsen voices a similar criticism. (page 3$)^{35}$ In such cases, how is it possible to draw a distinction between causal relations and non-causal associations? In order to meet this concern a further element must be added to the definition - a counterfactual.

\section{COUNTERFACTUALS}

A few statisticians and epidemiologists have advocated a counterfactual definition of causation. ${ }^{46-48}$ A counterfactual statement draws a contrast between one outcome given certain conditions and another outcome given alternative conditions. For example, "if an hour ago I had taken two aspirins instead of just a glass of water, my headache would now be gone." Thus, Rubin defines the causal effect of treatment $\mathrm{T}$, compared to absence of treatment, as the outcome given $\mathrm{T}$ minus the outcome given the absence of $T^{47}$ The definition requires a ceteris paribus condition that everything else is held constant.

Counterfactuals can be either deterministic or probabilistic. The aspirin counterfactual can be revised to read: "If an hour ago I had taken two aspirins instead of just a glass of water, I 
would be much less likely to still have a headache." Some recent philosophical accounts of probabilistic causation incorporate counterfactuals. ${ }^{3940434}$ For example, under Cartwright's definition ${ }^{43} \mathrm{C}$ causes $\mathrm{E}$ if the probability of $\mathrm{E}$ given $\mathrm{C}$ is greater than the probability of $\mathrm{E}$ given the absence of $\mathrm{C}$, while all else is held constant.

A counterfactual definition is not inconsistent with the necessary cause, sufficientcomponent cause, or probabilistic cause definitions. Rather, counterfactuals articulate an additional attribute that we suspect will enhance any definition of causation by strengthening the distinction between causation and mere correlation. While some commentators have emphasised the role of counterfactuals more than others, none have argued that causes do not behave according to counterfactuals. Nevertheless, the counterfactual definition is insufficient by itself as a definition of causation. While the counterfactual definition essentially states that the presence or absence of the cause "makes a difference", the necessary and sufficient, sufficient-component, and probabilistic definitions clarify what kind of difference it must make; on the probabilistic view a cause makes a difference in the chance of the effect.

The ceteris paribus condition must be interpreted carefully, however. While the ceteris paribus condition is key to distinguishing causation from non-causal associations in theory, it is not intended to be met in practice. Indeed, the condition is rarely met in actual scientific research; that is, the same individual cannot be observed in the exact same circumstances as both as a smoker and a non-smoker. ${ }^{46}$ Karhausen erroneously objects that a probabilistic definition as outlined by philosophers of science $^{43}$ is too strict for epidemiological practice, because it is defined through a ceteris paribus condition. ${ }^{12}$

This confusion between criteria for defining a cause (ontology) and criteria for identifying a cause empirically (epistemology) should be avoided. Some epidemiologists have failed to make this distinction, including intervention as part of a definition of what a cause is ${ }^{46}$ or referring to observed relative frequencies in stating a probabilistic definition of causation. (page 263) 18 $^{35}$ But a definition of cause should not depend on what differences have been measured or whether a particular intervention has been attempted. Rather, the ceteris paribus condition is hypothetical (that is, the contaminated Broad Street well is a cause of the cholera epidemic when it is the case that if the Broad Street pump was shut down, then cholera incidence would decrease). Extra care is required in the context of counterfactuals because they are often used not only in defining causation but also in constructing causal models for scientific inference..$^{51}$

We argue that the probabilistic definition combined with a counterfactual condition, like that offered by Cartwright, provides the greatest promise for epidemiology. It is consistent with both deterministic and probabilistic causal models, treating deterministic models as a limiting or extreme case. Thus, it also makes fewer assumptions about unobserved natural phenomena, removing the need to always posit hidden deterministic component causes. While a causal model of a specific phenomenon may posit hidden mechanisms, a definition requires generalisability and should make few assumptions. So why do some commentators continue to resist the probabilistic definition? Different views about the role of epidemiology relative to other sciences and to the practice of public health influence choices about causal models. In the following two sections, we explain how challenges in defining causation are related to two current debates in epidemiology.

\section{Causation at many levels}

Is there a hierarchy of causation at different levels of observation? According to the discussion so far, there is no reason to assert that causes at one level, such as molecules, are any more real or significant than causes at another level, such as social factors. Yet the priority of different levels of observation has been a topic of debate in the epidemiological literature. In 1973 Susser $^{20}$ described the importance of recognising different levels of organisation in evaluating causal hypotheses. During the past 15 years, much ink has been spilled over the value of "black box" strategies for investigating causal relations. ${ }^{52-54}$ Critics of "black box" epidemiology argued that scientific understanding was only advanced by looking inside the black box to understand the underlying mechanisms connecting cause and effect at the biological level. ${ }^{556}$ More recently, advocates of the importance of social factors in disease have argued that causal phenomena at the social level cannot be fully reduced to biology or to individual behaviours, like smoking. ${ }^{57-60}$ For example, characteristics of the quality of a neighbourhood may influence health outcomes at the individual level, such as cases of gonorrhea ${ }^{61}$; these neighbourhood characteristics cannot be captured by looking solely at characteristics of individual residents (such as income). This debate has substantial import for the allocation of resources in research and intervention.

What does this have to do with the different notions of causation discussed? In short, the positions in the black box debate are polarised along similar lines as those in discussion of different definitions of causation (particularly deterministic versus probabilistic). Advocates of the strict necessary cause view insist that causes should be identified at the level of internal biological mechanisms (rather than in behavioural or social factors). ${ }^{2122}{ }^{24}$ And those who advocate a prominent role for social and group level effects ${ }^{8762}$ are also critical of the sufficient-component cause and necessary cause definitions, urging instead a broader category of causation.

These differences correspond, in turn, to different types of scientific explanations. In developing a causal explanation of an observed association, there is a popular tendency among both lay persons and scientists to prioritise knowledge at the molecular level. ${ }^{63}$ In the 
1950s, for instance, sceptics doubted whether cigarette smoking patterns could provide a sufficient explanation of lung cancer occurrence. Instead, they sought a highly specific necessary cause (a molecule within cigarette smoke) that would provide a one to one correlation with disease outcome, thus providing a seemingly more complete explanation. ${ }^{64}$ Lurking behind this belief are the assumptions that lung cancer occurrence can be fully reduced to and explained by causes at the molecular level and that those causes behave deterministically. Among biologists, however, this simple reductionism has been widely criticised. ${ }^{65}$ In contrast, Susser and Susser have recently called for epidemiological theory that unifies disparate phenomena, from causal pathways at the societal level to pathogenesis at the molecular level; these are multi-level explanations. ${ }^{66}$

Probabilistic causes are often charged with offering only incomplete explanations because they retain an irreducible element of chance. Mid-20th century philosophers of science argued that unlikely events could not be explained. ${ }^{67}$ But more recent scholars have rejected that view, urging that probabilistic causes offer partial explanations for particular outcomes. ${ }^{40}{ }^{41} 68$ This view more closely mirrors epidemiological thinking. However, in this context it is important, again, to distinguish between a satisfactory causal model and a definition of causation. In developing causal models scientists sometimes do seek to reduce the influence of chance as much as possible, as it may be inversely related to the model's explanatory power. While the claim that smoking causes lung cancer explains differences in lung cancer rates between groups, it does not explain why some smokers develop lung cancer and other do not. There is still hope that the latter can eventually be fully explained by deterministic molecular mechanisms. We have no objection to the use of deterministic models where appropriate. But a definition of causation must allow for the possibility that chance is inherent in some natural processes. The probabilistic definition of causation allows for construction of both deterministic and probabilistic models, which are essential to both the biological and social sciences.

\section{Practical versus scientific goals of epidemiology}

A number of commentators have drawn a distinction between a strict "scientific" or "logical" definition of causation and a more flexible practical definition. ${ }^{9} 123749$ They do so because they believe that the aims of science and public health are different. The primary aim of public health is "to intervene to reduce morbidity and mortality from the disease."(page 3$)^{2}$ In contrast, the primary aim of science is, as commonly stated, to explain the world; such investigations may or may not lead to effective public health strategies.

Thus, some have recommended that epidemiologists abandon the traditional scientific concept of causes as necessary and sufficient conditions in favour of a broader concept with more practical value. ${ }^{97}$ Bunge, the philosopher of science, is frequently cited in this context for his claim that there are more forms of determination than strict determinism. ${ }^{69}$ Even some advocates of determinism admit that while probabilistic causal models are not wholly accurate, they can provide quick and dirty descriptions of phenomena that may be useful. ${ }^{19}$ Thus, Rothman suggests that while an individual's risk of disease at time $\mathrm{T}$ must be either 1 or 0 , probabilistic risk statements can be viewed as statements about the likelihood of a sufficient cause being present.(page 589) ${ }^{26}$

Yet this practical notion of causation tends to be viewed as a lesser compromise to the more robust "scientific" notion of cause because of a lingering assumption of determinism. ${ }^{70}$ The unfortunate consequence is that the claims of epidemiology seem to be practical but not truly scientific; sceptics of epidemiological methods might wonder, if epidemiologists use an unscientific definition of causation, how can they be expected to identify genuine causes? Drawing a distinction between a "scientific" and a "practical" definition of causation further contributes to a longstanding tension within the discipline of epidemiology between allegiance to science and allegiance to public health. Some recent commentators have responded to this tension by restraining the boundaries of epidemiology and maintaining that the discipline best contributes to public health by maintaining scientific rigour and separating science from public health policy. ${ }^{71}$

However, we maintain that a strong and effective definition of causation for epidemiology must be consistent with the aims of both science and public health. Although epidemiologists may debate their proper role in public health, it is clear that epidemiology cannot be divorced from the application of its findings. While we argued earlier that a probabilistic view of causation is consistent with modern theories of biological science, here we note that it also has some distinct advantages for the application of causal knowledge in practical public health efforts.

For public health it is often not enough to know that " $x$ is part of a constellation of conditions sufficient for y." Public health efforts target particular causes chosen for practical reasons, ${ }^{72}$ rather than targeting Mill's total constellation of causes. Moreover, quantifying the relative contribution of different types of causes is important, but sufficient-component causes and necessary causes offer little help here. Rothman and Greenland note that it can be said for many diseases that they are $100 \%$ caused by genetics and $100 \%$ by environment, because the appropriate sufficient causes contain both genetic and environmental components. ${ }^{29}$ The influence of a necessary or sufficient cause cannot be measured in degrees; its effect is all or nothing. A probabilistic view of causation, however, explains how different causes can exert different degrees of influence on an effect by referring to the amount by which each contributing cause increases the probability of the effect.

Moreover, a probabilistic definition is already implicit in practical reasoning about 
causes in epidemiology and public health. What does a physician mean when she tells her patient that he can reduce his risk of developing lung cancer by giving up smoking? Does she mean that he might be one of those individuals for whom smoking might be the final component in a sufficient cause for lung cancer? The implication here is that if he only knew the status of the other component causes (that is, does he carry the right form of gene $x$ ?) he might not have to give up smoking after all. But this view trivialises the nature of public health advice. In practice, the clinician means that giving up smoking will actually lower that individual's probability of developing lung cancer-not lower it from 1 to 0 , but lower it significantly. The deterministic assumption of hidden effect modifiers is thus more likely to thwart than to help public health efforts. The underlying definition of causation used is important for other applications of epidemiological findings as well, such as in toxic tort cases; in these cases, an implicit assumption of deterministic causation has led some courts to apply unreasonable criteria for evaluating epidemiologic evidence. $^{73} 74$

\section{Conclusion}

We have drawn on both epidemiological and philosophical literature on causation, but philosophers and epidemiologists, of course, pursue very different objects of study. While philosophers are interested in general principles of causation, epidemiologists tend to be interested in particular examples of causal relations. Thus, philosophers seek definitions while epidemiologists construct causal models. However, we have argued that an epidemiologist's beliefs and assumptions about cause carry practical consequences, because causal models are constructed within limits set by that definition the epidemiologist has in mind (whether explicit or implicit). Thus, if a deterministic definition of causation is assumed, only deterministic models will be recognised as causal. Because causation is so central to what epidemiologists do, we argue that it is crucial that epidemiologists understand the implications of applying a particular definition of causation.

We argue for using a probabilistic definition of causation because it is more inclusive than the necessary and sufficient and sufficientcomponent cause definitions. Deterministic models can be treated as extreme cases of the probabilistic definition; thus, the probabilistic definition incorporates the concept of necessary and sufficient causes. In addition, there are some concepts, while insufficient for defining causation themselves, that cannot be dispensed with in any causal definition - counterfactuals, production, and temporality. Additionally, the probabilistic definition does not require assumptions about hidden biological mechanisms (that observed probabilistic phenomena always reduce to some underlying deterministic mechanism).

Nevertheless, a definition is not itself a theory of causation; there is more conceptual work to be done towards further specifying the nature of causation. Unfortunately, philosophical thinking about causation has been largely driven by the physical sciences, focusing on simple chains of events rather than the complex multi-level relations that make up biology. Thus, this is an area that needs further research. How are explanations at different levels, from the molecular to the social, related? And further inquiry is needed into the relation between concepts of causation and causal inference. Guidelines for drawing causal inferences may differ according to how causation is defined. Because causal inference is at the heart of what epidemiologists do, the theoretical study of causation has important consequences for the everyday practice of epidemiology.

The authors are grateful to Ellen Velie, Steve Goodman, Mervyn Susser, Michael S Kramer, Karen Goodman, Fred Suppe, and two anonymous referees for discussions and comments on earlier versions of this manuscript.

Funding: none.

Conflicts of interest: none.

1 Olsen J. Some consequences of adopting a conditional deterministic causal model in epidemiology. Eur f Public Health 1993;3:204-9.

2 MacMahon B, Pugh T. Epidemiology: principles and methods. Boston: Little, Brown, 1970.

3 Kramer MS, Lane DA. Causal propositions in clinical research and practice. F Clin Epidemiol 1992;45:639-49.

4 Krieger N, Zierler S. The need for epidemiologic theory. Epidemiology 1997;8:212-14

5 Susser M, Susser E. Choosing a future for epidemiology: I. Eras and paradigms. Am f Public Health 1996;86:668-73.

6 Nijhuis HGJ, Van Der Maesen LJG. The philosophical foundations of public health: an invitation to debate. $\mathcal{F}$ Epidemiol Community Health 1994;48:1-3.

7 MacMahon B, Pugh TF, Ipsen J. Epidemiologic methods. Boston: Little, Brown, 1960 .

8 Krieger N. Epidemiology and the web of causation: has anyone seen the spider? Soc Sci Med 1994;39:887-903.

9 Susser M. What is a cause and how do we know one? $A m \mathcal{F}$ Epidemiol 1991;133:635-48.

10 Hume D. An enquiry concerning human understanding. Indianapolis: Hackett, 1977.

1 Russell B. On the notion of cause. In: Mysticism and logic. New York: W W Norton, 1929.

12 Karhausen LR. The logic of causation in epidemiology. Scand 7 Soc Med 1996;24:8-13.

13 Renton A. Epidemiology and causation: a realist view. $\mathcal{f}$ Epidemiol Community Health 1994;48:79-85.

4 Gordis L. Epidemiology. 2nd ed. Philadelphia: W B Saunders, 2000.

15 Elwood M. Causal relationships in medicine: a practical system for critical appraisal. Oxford: Oxford University Press, 1988.

6 Timmreck TC. An introduction to epidemiology. Boston: Jones and Bartlett, 1994.

17 Last JMA. Dictionary of epidemiology, 2nd ed. New York: Oxford University Press, 1988

18 Lilienfeld DE, Stolley PD. Foundations of epidemiology. 3rd ed. New York: Oxford University Press, 1994.

19 Kleinbaum DG, Kupper LL, Morgenstern HL. Epidemiologic research: principles and quantitative methods. Belmont, CA: Lifetime Learning Publications, 1982

20 Susser M. Causal thinking in the health sciences: concepts and strategies of epidemiology. New York: Oxford University Press, 1973.

21 Stehbens WE. The concept of cause in disease. 7 Chronic Dis 1985;38:947-50.

22 Charlton BG. Attribution of causation in epidemiology: chain or mosaic? f Clin Epidemiol 1996;49:105-7.

23 Carter KC. Koch's postulates in relation to the work of Jacob Henle and Edwin Klebs. Med Hist 1985;29:353-74.

24 Bernard C. An introduction to the study of experimental medicine. (Translated by Henry Copley Greene). New York: Dover, 1957.

25 Evans AS. Causation and disease: a chronological journey. New York: Plenum, 1993.

26 Rothman KJ. Causes. Am f Epidemiol 1976;104:587-92.

27 Mill JS. Philosophy of scientific method. New York: Hafner, 1950 [1843].

28 Mackie JL. The cement of the universe: a study of causation. Oxford: Clarendon Press, 1974.

29 Rothman K, Greenland S. Modern epidemiology. 2nd ed. Philadelphia: Lippincott Williams and Wilkins, 1998.

30 Fisher RA. Indeterminism and natural selection. Philosophy of Science 1934;1:99-117.

31 Mayr E. Cause and effect in biology. Science 1961;134: 1501-6. 
32 Koopman JS, Weed DL. Epigenesis theory: a mathematical model relating causal concepts of pathogenesis in individuals to disease patterns in populations. Am F Epidemiol 1990 132:366-90.

33 Hogan MD, Kuper LL, Most BM, et al. Alternatives to Rothman's approach for assessing synergism (or antagonism) in cohort studies. Am F Epidemiol 1978;108:60-7.

34 Koopman JS. Causal models and sources of interaction. $A m$ f Epidemiol 1977;106:439-44.

35 Olsen J. Causes and prevention. Scand $\mathcal{f}$ Soc Med 1991;19:1-6.

36 Schlesselman JJ. "Proof" of cause and effect in epidemiologic studies: criteria for judgement. Prev Med 1987;16: 195-210.

37 International Agency for Research on Cancer. Cancer: causes, occurrences and control. IARC Scientific Publications no. 100. Lyon, France: International Agency for Research on Cancer, 1990

38 Suppes P. A probabilistic theory of causality. Amsterdam: North-Holland Publishing Company, 1970

39 Salmon WC. Scientific explanation and the causal structure of the world. Princeton: Princeton University Press, 1984.

40 Humphreys P. The chances of explanation: causal explanation in the social, medical, and physical sciences. Princeton: Princin the social, medical, and physic

41 Mellor DH. The facts of causation. London: Routledge, 1995.

42 Eells E. Probabilistic causality. Cambridge: Cambridge University Press, 1991.

43 Cartwright N. Nature's capacities and their measurement. Oxford: Clarendon Press, 1989.

44 Lewis D. B. Chancy causation. Postscript to causation. In Lewis D. Philosophical papers. Vol II. New York: Oxford University Press, 1986.

45 Cox DR. Causality: some statistical aspects. $\mathcal{F} R$ Stat $\operatorname{Soc} A$ 1992;155:291-301.

46 Holland PW. Statistics and causal inference. 7 Am Stat Assoc 1986;81:945-60.

47 Rubin DB. Estimating causal effects of treatments in randomized and nonrandomized studies. Fournal of Educational

48 Maldonado G, Greenland, S. Estimating causal effects. [abstract]. Presented at the Society for Epidemiologic Research 31st Annual Meeting, Chicago, Illinois, June, 1998. Am F Epidemiol 1998;147:S80.

49 Riegelman R. Contributory cause: unnecessary and insufficient. Postgrad Med 1979;66:177-9.

50 Pearl J. Causality: models, reasoning, and inference. Cambridge: Cambridge University Press, 2000

51 Cook TD, Campbell DT. Quasi-experimentation: design and analysis issues for field settings. Boston: Houghton Mifflin, 1979 .

52 Weed DL. Beyond black box epidemiology. Am f Public Health 1998;88:12-14.

53 Savitz DA. In defense of black box epidemiology. Epidemiology 1994;5:550-2.
54 Peto $\mathrm{R}$. The need for ignorance in cancer research. In: Duncan $\mathrm{R}$, Weston-Smith $\mathrm{M}$, eds. The encyclopedia of medical ignorance. Oxford: Pergamon Press, 1984:129-33.

55 Vandenbroucke JP. Is "The causes of cancer" a miasma theory for the end of the twentieth century? Int $\mathcal{f}$ Epidemiol 1988;17:708-9.

56 Skrabanek P. The emptiness of the black box. Epidemiology 1994;5:553-5.

57 Diez-Roux AV. Bringing context back into epidemiology: variables and fallacies in multilevel analysis. Am F Public Health 1998;88:216-22.

58 McMichael AJ. Prisoners of the proximate: loosening the constraints on epidemiology in an age of change. Am $\mathcal{F}$ Epidemiol 1999;149:887-97.

59 Fullilove RE, Edgoose JC, Fullilove MT. Chaos, criticality, and public health. 7 Natl Med Assoc 1997;89:311-16.

60 McMichael AJ. Prisoners of the proximate: loosening the constraints on epidemiology in an age of change. Am f Epidemiol 1999;149:887-97.

61 Cohen D, Spear S, Scribner R, et al. "Broken windows" and the risk of gonorrhea. Am f Public Health 2000;90:230-6.

62 Koopman JS, Lynch JW. Individual causal models and population system models in epidemiology. Am 7 Public Health 1999;89:1170-4.

63 Stout D. Direct link found between smoking and cancer. The New York Times 1996 Oct 18; section A: 1 (col 3).

64 Yerushalmy J, Palmer CE. On the methodology of investigations of etiologic factors in chronic diseases. F Chronic Dis 1959;10:27-40.

65 Mayr E. The growth of biological thought: diversity, evolution, and inheritance. Cambridge, MA: Belknap Press, 1982.

66 Susser M, Susser E. Choosing a future for epidemiology: II. From black box to Chinese boxes and eco-epidemiology. Am f Public Health 1996;86:674-7.

67 Hempel CG. Aspects of scientific explanation and other essays in the philosophy of science. New York: Free Press, 1965.

68 Schaffner KF. Discovery and explanation in biology and medicine. Chicago: University of Chicago Press, 1994

69 Bunge M. Causality and modern science. 3rd ed. New York: Dover, 1959.

70 Parascandola M. Epidemiology: second rate science? Public Health Rep 1998;113:312-23.

71 Savitz DA, Poole C, Miller WC. Reassessing the role of epidemiology in public health. Am 7 Public Health 1999;89: $1158-61$.

72 Hart HLA, Honoré T. Causation in the law. 2nd ed. Oxford: Clarendon Press, 1985.

73 Parascandola $M$. What is wrong with the probability of causation? furimetrics 1998;39:29-44.

74 Beyea J, Greenland S. The importance of specifying the underlying biologic model in estimating the probability of causation. Health Phys 1999;76:269-74. 